\title{
Seeking for the Leading Actor on the Cosmic Stage: Galaxies versus Supermassive Black Holes
}

\author{
Angela Bongiorno, ${ }^{1,2}$ Francesco Shankar, ${ }^{3}$ Francesca Civano, ${ }^{4}$ \\ Isabelle Gavignaud, ${ }^{5}$ and Antonis Georgakakis ${ }^{1}$ \\ ${ }^{1}$ Max-Planck-Institut für Extraterrestrische Physik (MPE), Giessenbachstraße 1, Garching bei, 85748 München, Germany
${ }^{2}$ Osservatorio Astronomico di Roma, Via Frascati 33, Monteporzio Catone, 00040 Rome, Italy
${ }^{3}$ GEPI, Observatoire de Paris, CNRS, Université Paris Diderot, 5 Place Jules Janssen, 92195 Meudon, France
${ }^{4}$ Smithsonian Astrophysical Observatory, 60 Garden Street, MS 67, Cambridge, MA 02138, USA
${ }^{5}$ Departamento de Ciencias Fisicas, Facultad de Ingenieria, Universidad Andres Bello, Avandia Republica 252 Santiago, Chile
}

Correspondence should be addressed to Angela Bongiorno, angela.bongiorno@oa-roma.inaf.it

Received 31 May 2012; Accepted 31 May 2012

Copyright (C) 2012 Angela Bongiorno et al. This is an open access article distributed under the Creative Commons Attribution License, which permits unrestricted use, distribution, and reproduction in any medium, provided the original work is properly cited.

A major development in extragalactic astrophysics in recent years has been the realization that active galactic nuclei (AGN), which signpost accretion events onto supermassive black holes (SMBHs) $[1,2]$, may play a fundamental role in the formation and evolution of galaxies. Understanding the physics that drive the growth of $\mathrm{SMBH}$ across cosmic time is therefore important for having a complete picture of galaxy formation. The two processes of galaxy and $\mathrm{BH}$ evolution can no longer be regarded as separate, as was the case until about 10 years ago, but need to be studied in conjunction.

One of the first indications for a correlation between the formation of galaxies and the growth of SMBHs at their centres is the striking similarity between the redshift evolution of the accretion density and the star-formation rate density $[3,4]$ of the Universe. Both quantities show a rapid increase from the local Universe to $z \approx 1$, followed by a broad plateau at $\mathrm{z} \approx 2-4$ and a decline at $z \geq 4$, although the high redshift behavior of X-ray AGN remains controversial [5].

These similarities are more than a mere coincidence or a manifestation of the fact that the Universe was overall more active in the past. Observational evidence now shows that most, if not all, galaxies in the local universe posses a central $\mathrm{SMBH}$ at their center. Moreover, there is now emerging consensus from dynamical observations that SMBHs at the center of the local massive and bulge-dominated galaxies are tightly correlated with the velocity dispersion and masses of their stellar hosts, with an intrinsic scatter of a factor of two or even less [6-8]. Such strong correlations argue for a physical association between the two processes and suggest that BHs must have evolved, or coevolved, with their stellar hosts at some point in their past.

Analytical calculations identify AGN feedback as the process that can potentially link SMBH growth and star formation (e.g., [9-14]). In this picture, the energy released by the AGN is sufficient to either heat up or blow away the cold gas of galaxies, thereby irreversibly altering their evolution. Observations of powerful and/or nearby active SMBHs have recently started finding evidence for outflows, most likely associated with the AGN, in either the warm (e.g., $[15])$ or the cold (e.g., $[16,17])$ gas component of their host galaxies.

Despite the increasing evidence for the importance of $\mathrm{SMBH}$ growth in galaxy formation, we have just started exploring the physical processes at play. The nature of AGN feedback, how it is related to the fueling mode of the SMBH, and ultimately what is its impact on kpc or even Mpc scales are still debated.

To shed light on these issues from both the observational and theoretical points of view, we initiated this special issue (http://www.hindawi.com/journals/aa/si/610485/) which collects reviews and new results, contributed by some of the key researchers in the field. We made an effort to 
bring together observers and theoreticians to provide the community with a comprehensive state-of-the-art overview of our understanding of SMBH evolution across cosmic time. In this respect, the special issue deals with topics from the smallest to the largest scales including, for example, $\mathrm{BH}$ accretion mechanisms and properties, the influence on their host galaxies, and the connection to the dark matter halos. We hope this special issue will become a useful reference for all researchers in the field.

The special issue starts with a paper from S. Bianchi et al. who discuss the recent developments on the AGN unified models. They review the standard unified model to then move to an updated unification scenario that can better explain the complex phenomenology observed. This paper is followed by a series of papers which constitute a comprehensive description of the accretion history of the Universe. In particular, F. Fiore et al. present the $z>3$ $\mathrm{X}$-ray sources number counts in the $0.5-2 \mathrm{keV}$ band and make predictions for new missions; E. Treister and C. M. Urry review what we know about the energy output of AGN describing the most common way to isolate these sources at different wavebands. They also summarize the cosmic history of black hole accretion, i.e., when in the history of the Universe supermassive black holes were obtaining most of their mass. B. C. Kelly and A. Merloni present the demographic of SMBHs in the local Universe discussing advantages and disadvantages of the different methods for estimating the black hole mass function, and A. Sesana reviews the current understanding of massive $\mathrm{BH}$ formation and evolution along the cosmic history highlighting which future observations will help to shed light on the cosmic history of SMBHs, paying particular attention to the upcoming gravitational wave window. A comprehensive discussion of current ideas of BHs fueling mechanisms is presented by G. Lodato, while T. Johannsen discusses possible new observational tests for confirming the existence of $\mathrm{BH}$ at the centers of galaxies.

However, $\mathrm{BHs}$ do not grow only via gas accretion but possibly also via BH-BH mergers. M. Dotti, A. Sesana et al. review the state of the art of mergers as an additional channel of BH growth including the expected observational signatures of massive binaries. In this context, S. Komossa presents the results of numerical relativity simulations which imply that after binary coalescence in a galaxy merger the newly formed single SMBH can receive kick velocities up to several $1000 \mathrm{~km} / \mathrm{s}$ due to anisotropic emission of gravitational waves. Moreover, she presents the observational signatures of recoiling SMBHs and the properties of the first candidates which have emerged.

After that, we devoted part of the special issue to our current understanding of the connection between SMBHs and their host galaxies inferred from a variety of observational probes. The latest results on the AGN/Starburst connection are presented by E. Sani and E. Nardini who studied a peculiar ultraluminous infrared galaxy (IRAS 20551-4250) experiencing an intense starburst but hosting a highly obscured AGN. A. Constantin and A. C. Seth present the peculiar nature of the nucleus of M94, which is the least luminous broad-line (type 1) LINER with possibly the least luminous broad line region known. This is followed by a paper from R. Decarli et al. on the extension of the scaling relation between SMBHs and host galaxies to the smallest observed local SMBHs, showing that the relation holds over 2 dex in both $M_{\mathrm{BH}}$ and $M_{*}$. Moreover, N. Neumayer \& C. J. Walcher and P. Erwin \& D. A. Gadotti extended the work to the nuclear star clusters and their embedded BHs, which are the possible precursors of massive black holes in galaxy nuclei. N. Neumayer \& C. J. Walcher studied the low mass end of the global-to-nucleus relations finding that the $M_{\mathrm{BH}}-M_{\text {bulge }}$ relation may well flatten at low masses while the $M_{\mathrm{BH}}-\sigma$ relation may steepen. Moreover, P. Erwin, \& D. A. Gadotti analyze a sample of disk galaxies and performing a $2 \mathrm{D}$ bulge/disk/bar decompositions show that while SMBHs correlate with the stellar mass of the bulge component of galaxies, the masses of nuclear star clusters correlate much better with the total galaxy stellar mass.

We conclude the special issue by discussing what we can learn from studying the large-scale environment of AGN. R. Fassbender et al. studied the distribution of X-ray AGN in the large-scale structure environments of $22 \mathrm{X}$-ray luminous galaxy clusters in the redshift range $0.9<z<1.6$. They found two overdensities, one at $\mathrm{r}<1 \mathrm{Mpc}$ of predominantly low-luminosity AGN and another one of brighter soft-band detected AGN at cluster-centric distances of 2-3 Mpc (about three times the average cluster radius $\mathrm{R}_{200}$ of the systems). Their results support the idea of two different physical triggering mechanisms of X-ray AGN activity in dependence of the radially changing large-scale structure environment of the distant clusters. M. Gitti et al. draw a qualitative picture of the current knowledge of the effects of the AGN feedback on the intracluster medium by summarizing the recent results in this field. Finally, N. Cappelluti et al. review the now abundant data on AGN small- and large-scale clustering properties from different deep and large surveys and the full important information we can derive from them.

Angela Bongiorno
Francesco Shankar
Francesca Civano
Isabelle Gavignaud
Antonis Georgakakis

\section{References}

[1] A. Merloni and S. Heinz, "A synthesis model for AGN evolution: supermassive black holes growth and feedback modes," Monthly Notices of the Royal Astronomical Society, vol. 388, no. 3, pp. 1011-1030, 2008.

[2] F. Shankar, "The demography of supermassive black holes: growing monsters at the heart of galaxies," New Astronomy Reviews, vol. 53, no. 4-6, pp. 57-77, 2009.

[3] A. Merloni, "The anti-hierarchical growth of supermassive black holes," Monthly Notices of the Royal Astronomical Society, vol. 353, no. 4, pp. 1035-1047, 2004.

[4] P. F. Hopkins, L. Hernquist, T. J. Cox, T. Di Matteo, B. Robertson, and V. Springel, "A unified, merger-driven model of the origin of starbursts, quasars, the cosmic X-ray background, supermassive black holes, and galaxy spheroids," The Astrophysical Journal Supplement Series, vol. 163, no. 1, pp. 149, 2006. 
[5] M. Brusa, A. Comastri, R. Gilli et al., "High-redshift quasars in the cosmos survey: the space density of $z>3 \mathrm{X}$-ray selected QSOs," The Astrophysical Journal, vol. 693, no. 1, article 8, 2009.

[6] J. Magorrian, S. Tremaine, D. Richstone et al., "The demography of massive dark objects in galaxy centers," The Astronomical Journal, vol. 115, no. 6, pp. 2285-2305, 1998.

[7] L. Ferrarese and D. Merritt, "A fundamental relation between supermassive black holes and their host galaxies," The Astrophysical Journal, vol. 539, pp. L9-L12, 2000.

[8] N. Häring and H.-W. Rix, "On the black hole mass-bulge mass relation," The Astrophysical Journal, vol. 604, no. 2, pp. L89L92, 2004.

[9] J. Silk and M. J. Rees, "Quasars and galaxy formation," Astronomy \& Astrophysics, vol. 331, pp. L1-L4, 1998.

[10] A. C. Fabian, "The obscured growth of massive black holes," Monthly Notices of the Royal Astronomical Society, vol. 308, no. 4, pp. L39-L43, 1999.

[11] A. King, "Black holes, galaxy formation, and the $M_{\mathrm{BH}}-\sigma$ relation," The Astrophysical Journal Letters, vol. 596, no. 1, article L27, 2003.

[12] G. L. Granato, G. de Zotti, L. Silva, A. Bressan, and L. Danese, "A physical model for the coevolution of QSOs and their spheroidal hosts," The Astrophysical Journal, vol. 600, no. 2, pp. 580-594, 2004.

[13] D. J. Croton, V. Springel, S. D. M. White et al., "The many lives of active galactic nuclei: cooling flows, black holes and the luminosities and colours of galaxies," Monthly Notices of the Royal Astronomical Society, vol. 365, no. 1, pp. 11-28, 2006.

[14] N. Menci, A. Fontana, E. Giallongo, A. Grazian, and S. Salimbeni, "The abundance of distant and extremely red galaxies: the role of AGN feedback in hierarchical models," The Astrophysical Journal Letters, vol. 647, no. 2, pp. 753-762, 2006.

[15] J. Holt, C. N. Tadhunter, R. Morganti, and B. H. C. Emonts, "The impact of the warm outflow in the young (GPS) radio source and ULIRG PKS 1345+12 (4C 12.50)," Monthly Notices of the Royal Astronomical Society, vol. 410, no. 3, pp. 15271536, 2011.

[16] C. Feruglio, R. Maiolino, E. Piconcelli et al., "Quasar feedback revealed by giant molecular outflows," Astronomy \& Astrophysics, vol. 518, no. 13, article L155, 2010.

[17] E. Sturm, E. González-Alfonso, S. Veilleux et al., "Massive molecular outflows and negative feedback in ULIRGs observed by HERSCHEL-pacs," The Astrophysical Journal Letters, vol. 733, no. 1, article L16, 2011. 

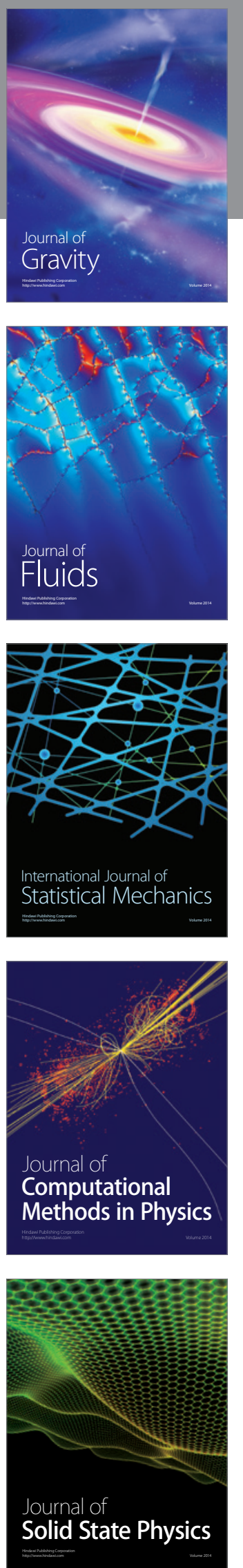

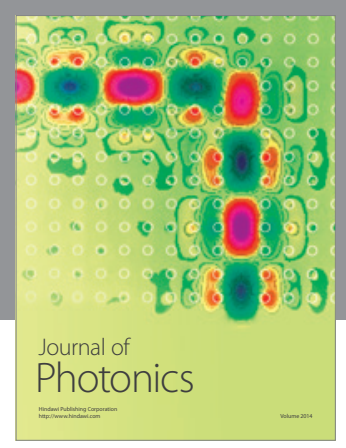

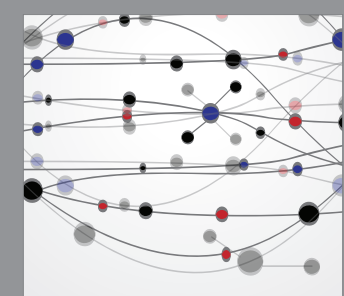

The Scientific World Journal
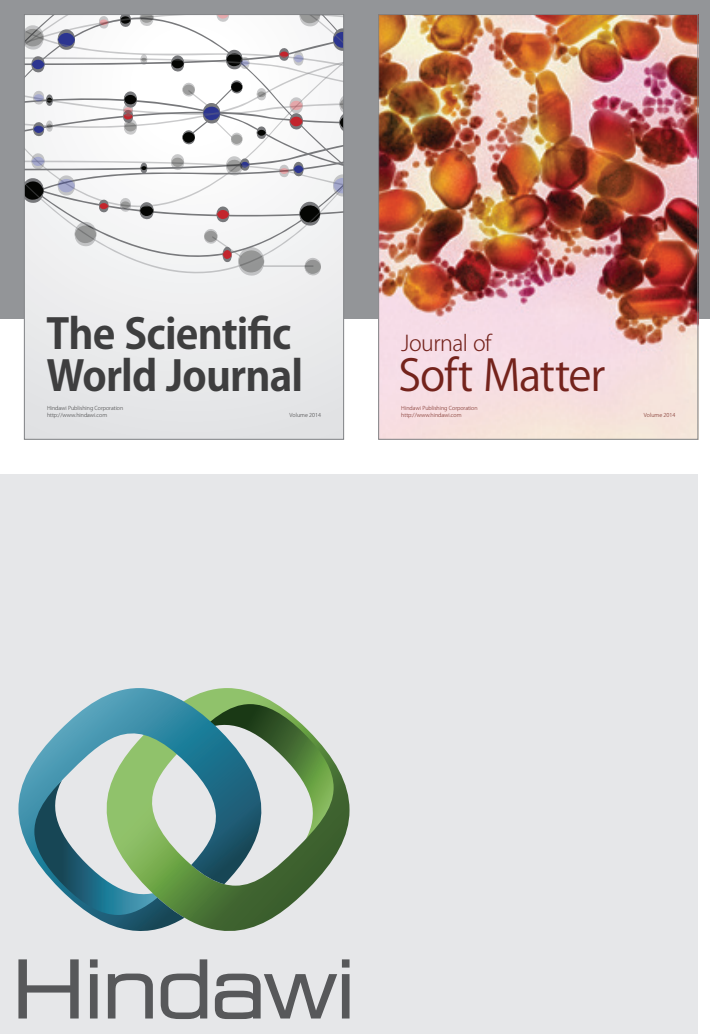

Submit your manuscripts at

http://www.hindawi.com
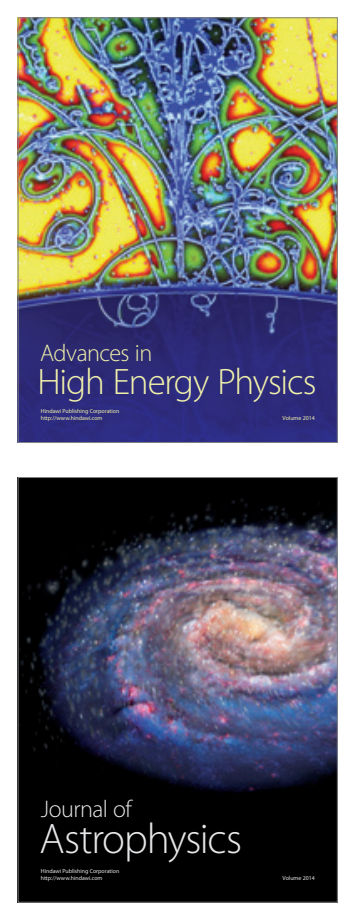
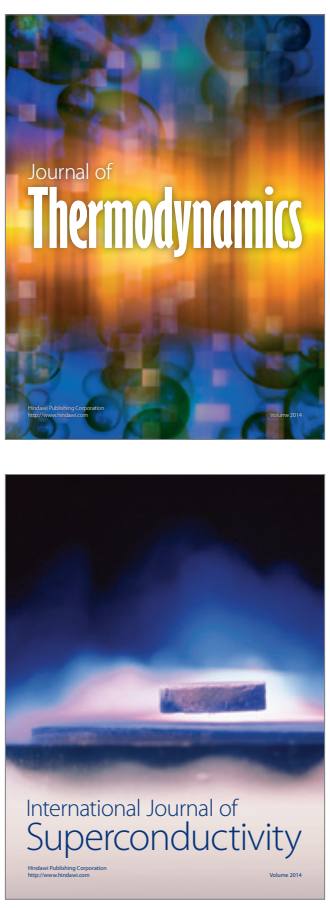
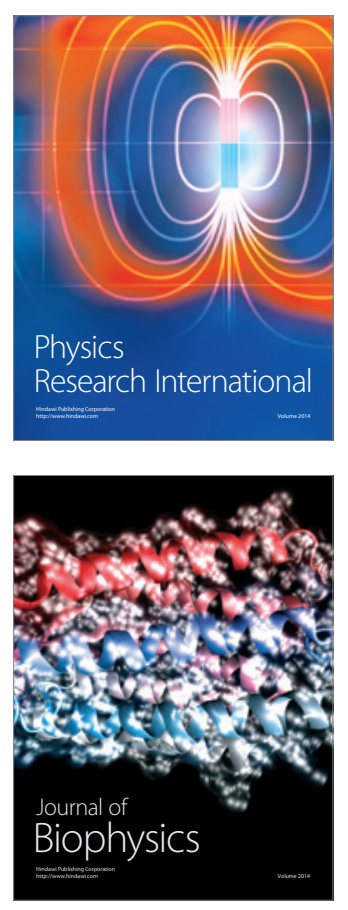
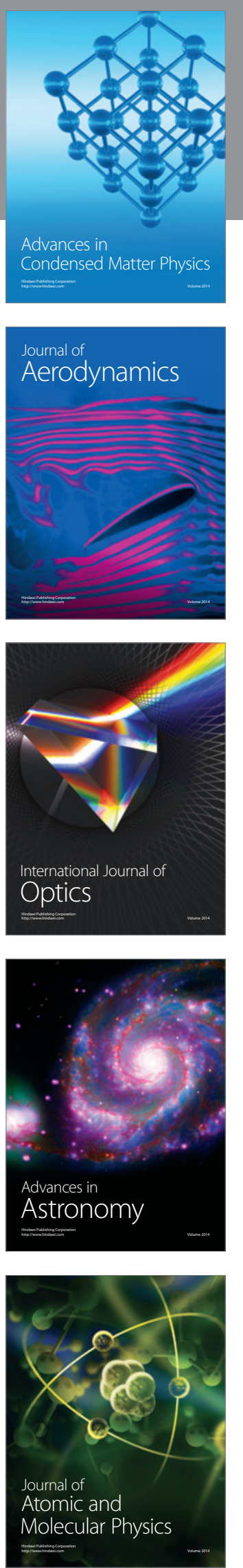